\title{
Evaluation of the performance of three different methods used in the identification of rigid body properties
}

\author{
R.A.B. Almeida ${ }^{\mathrm{a}}$, A.P.V. Urgueira $^{\mathrm{a}}$ and N.M.M. Maia ${ }^{\mathrm{b}, *}$ \\ ${ }^{a}$ Department of Mechanical Engineering and Industrial Production, New University of Lisbon, 2829-516 Monte de \\ Caparica, Portugal \\ ${ }^{\mathrm{b}}$ Department of Mechanical Engineering, Instituto Superior Técnico, Technical University of Lisbon, Av. Rovisco \\ Pais, 1049-001 Lisboa, Portugal
}

\begin{abstract}
The identification of the rigid body properties of a structure is an important matter in various structural dynamic applications, namely in structural modification (coupling/uncoupling), optimization and vibration control. In most situations, the experimental route is the only via to obtain the desired dynamic properties. This goal is achieved by an inverse process which starts from the measurement of Frequency Response Functions (FRFs) and ends on matrices describing the mass distribution of a rigid body.

The objective of the present article is the evaluation and comparison of the performance of three different kinds of identification methods that share a common characteristic - they all use FRFs as inputs to the inverse process. One of them, the Inertia Restraint Method (IRM) makes use of the accelerance FRF (information condensed at zero frequency), while the other two methods make use of receptance FRFs as inputs, although in different manners; in one of them the properties are evaluated directly from the measurement data - Direct Physical Parameter Identification Method (DPPIM) - whereas the other type requires a modal identification procedure as an intermediate step - Modal Method (MM). An actual experimental structure forms the case study to illustrate the performance of the three different techniques.
\end{abstract}

Keywords: Rigid body properties, modal analysis

\section{Nomenclature}

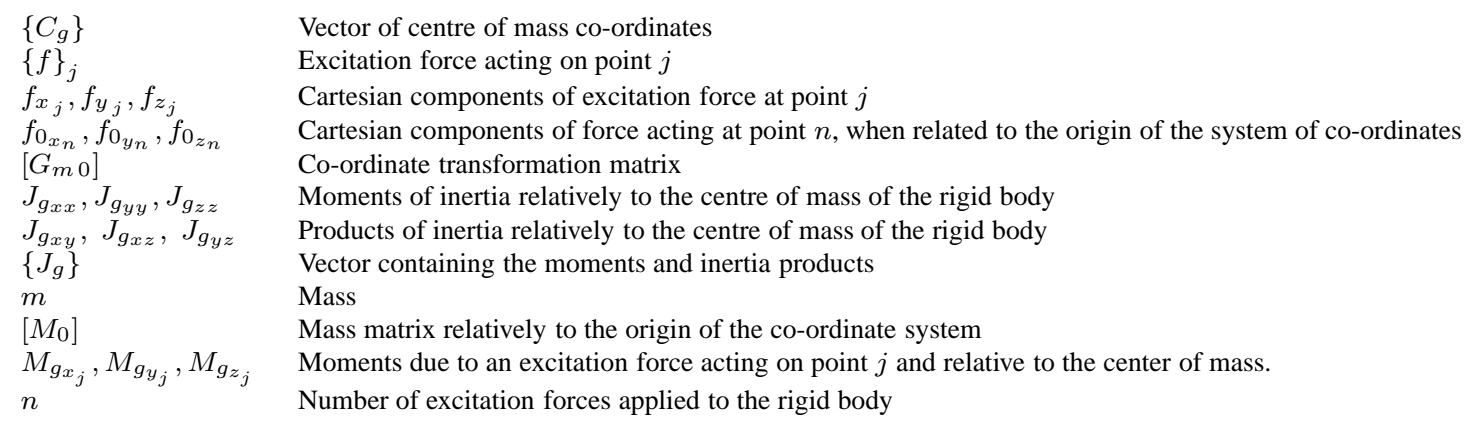

${ }^{*}$ Corresponding author. E-mail: nmaia @ dem.ist.utl.pt. 


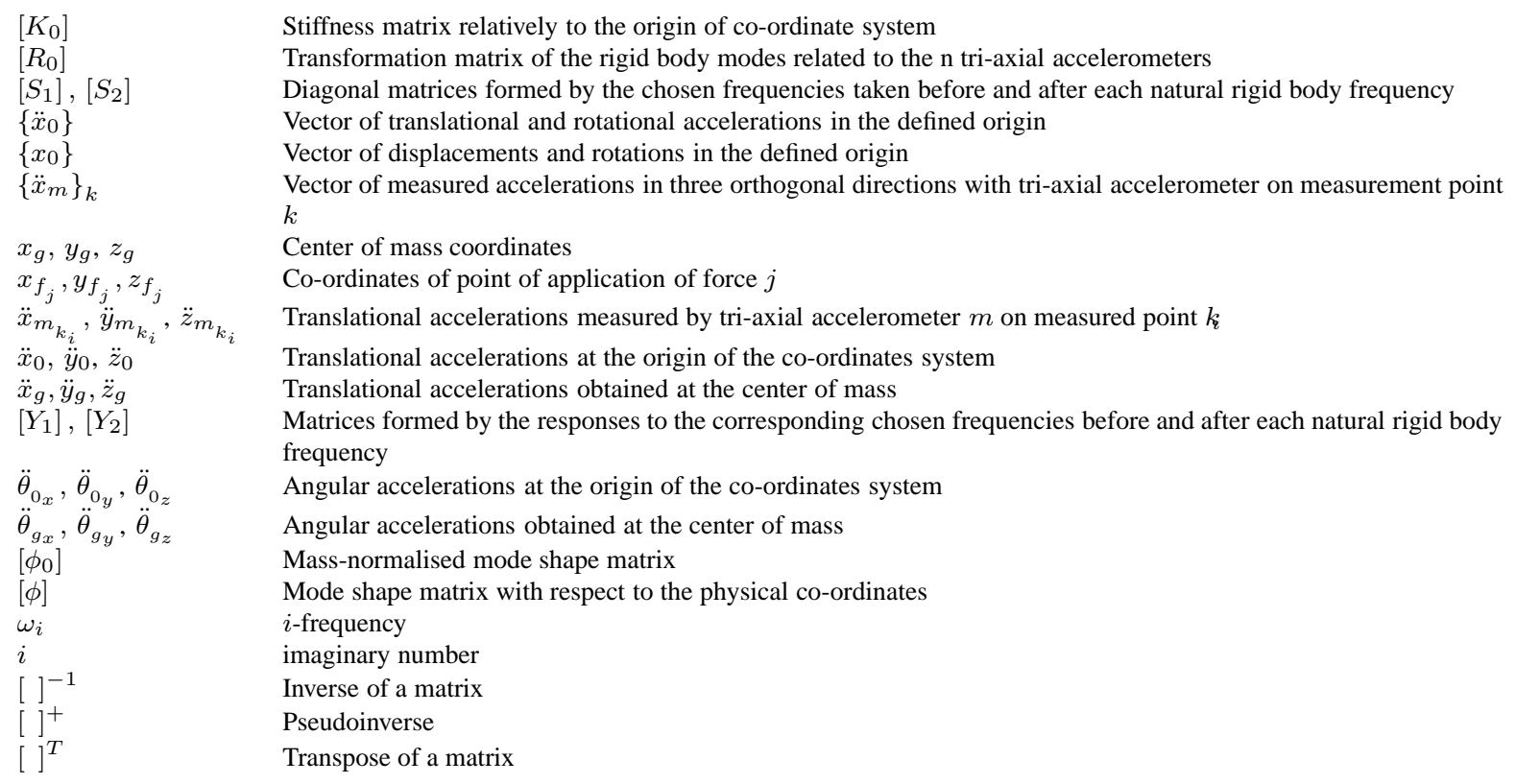

\section{Introduction}

The determination of the Rigid Body Inertia Properties (RBIP) of a given structure may reveal some difficulties whenever its theoretical model does not exist, or when modifications are made to the original structure. In these cases the establishment of an accurate theoretical model can be very time consuming. The alternative may reside on the use of experimental data readily available from measurements.

The methods that use experimental data could be primary classified as static or dynamic methods [1]. The former class of methods only allow, at most, the evaluation of four (mass and three co-ordinates of centre of mass) of the ten inertia properties of the rigid body (the other six form the inertia tensor).

The dynamic methods are frequently separated into two main categories: Time Domain Methods (TDM) and Frequency Domain Methods (FDM). The early TDM to mention are the classical pendulum methods. Most recently, some authors [2,3] developed variations of the TDM, based on the evaluation of vibration test data. The advantage of these methods is the direct evaluation of the test data in the time domain. A disadvantage is that, if the system under observation does not behave like a rigid body in the excited frequency range, low pass filtering of the test data must be performed. Frequency Domain Methods can circumvent this drawback because it is possible to separate the rigid and elastic system behaviour, even if the first elastic natural frequency is very low. The FDM will be the main subject of the present work. These methods can be divided into three groups: Inertia Restraint Methods (IRM), Modal Methods (MM) and Direct Physical Parameter Identification Method (DPPIM). The IRM have been widely studied and they stand on the principle that the dynamic response of freely supported structures is characterized in the low frequency domain by a constant term designated as "mass line (ML)". Additionally the IRM methods can be grouped into two main categories: Iterative Methods (IM) [4,5] and Direct Methods (DM) [6-9,24], according to the way that RBIP are obtained. The first group assumes the knowledge of one or several properties and, by an iterative process, the remainders are calculated. The DM group use directly and solely the data extracted from measured FRFs. The MM are based on the orthogonality relationship between the mass matrix and the rigid body modes [14-17]. The main characteristic of these methods is that they also use the results from a modal identification of the FRFs obtained from test on a quasi-free system. Some investigators refer that a disadvantage of these methods is that, in general, not all the rigid body modes can be excited in an actual experimental test. A group of investigators [11,19] are devoting the attention to the development of indicators that provide not only information about the number of rigid body modes that are excited in an experimental test, but also to the level of excitation of each rigid body mode. Recently, new methodologies $[18,26]$ using the principles of MM and structural modification procedures have been presented. Contrary to the IRM and MM the DPPIM use directly the measured FRFs, allowing for the determination of mass, 
stiffness of supports and damping parameters. Some authors have focused their attention on these methods $[10,12$, 13]. Some investigators [20], have used a similar approach to the DPPIM although incorporating separately the acceleration and force date.

Some investigators have tested the effectiveness of various methods [21-24]. In this work a further evaluation is carried out on the performance of three methods, all of them using the FRFs measured on an actual structure.

\section{Theory}

In the present work the determination of the ten inertia parameters follows closely, the IRM method presented by Almeida [24], the MM methods developed by Conti and Bretl [14] and Toivola and Nuutila [15] and the DPPIM method presented by Huang and Lallement [13].

\subsection{Inertia Restraint Method (IRM)}

In this work an IRM method proposed by Almeida [24] is discussed. This method uses as input the measured accelerations or residual mass values collected at different locations due to artificial excitations produced at various points in a softly supported structure. There is a similarity with other works [8,25], although these methods require a previous knowledge of the mass value. In contrast to other methods $[6,7,9]$ the ten rigid body properties are obtained in two distinct steps; in a first step the mass and centre of mass co-ordinates are calculated and in a second step the moments and inertia products are obtained.

\subsubsection{First Step}

Let us consider the acceleration at $k$ points on the structure due to an excitation force. If the measurements at these points are made using tri-axial accelerometers they all can be grouped in a response vector $\left\{\ddot{x}_{m}\right\}_{\text {total }}$ that can be related to the responses at an arbitrary origin $\left\{\ddot{x}_{0}\right\}$ by the following equation:

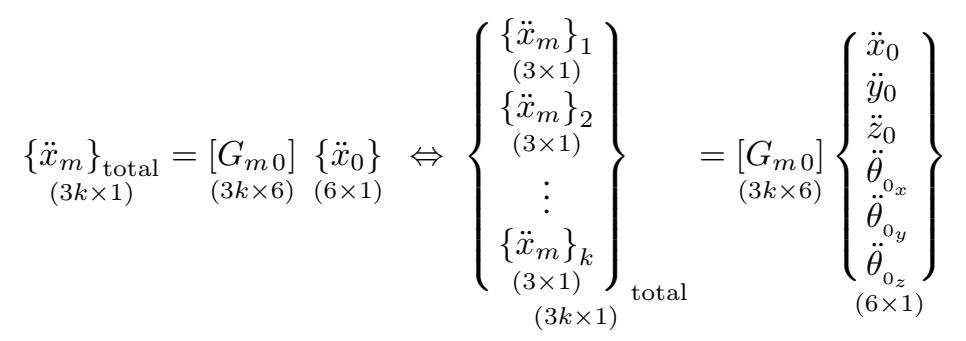

where $\left[G_{m 0}\right]$ corresponds to a co-ordinate transformation matrix [24]. Therefore,

$$
\begin{aligned}
& \left\{\begin{array}{l}
\left.\ddot{x}_{0}\right\} \\
(6 \times 1)
\end{array}=\underset{(6 \times 3 k)}{\left[G_{m 0} 0\right.}\right]^{+} \underset{(3 k \times 1)}{\left\{\ddot{x}_{m}\right\}_{\text {total }}} \\
& {\left[G_{m 0}\right]^{+}=\left(\begin{array}{ll}
{\left[G_{m 0}\right]^{T}\left[G_{m 0}\right]} & )^{-1}\left[G_{m 0}\right]^{T}\left\{\ddot{x}_{m}\right\}_{\text {total }}
\end{array} .\right.}
\end{aligned}
$$

The various response data at the origin due to the excitation forces applied at $n$ points on the structure, can be interrelated as;

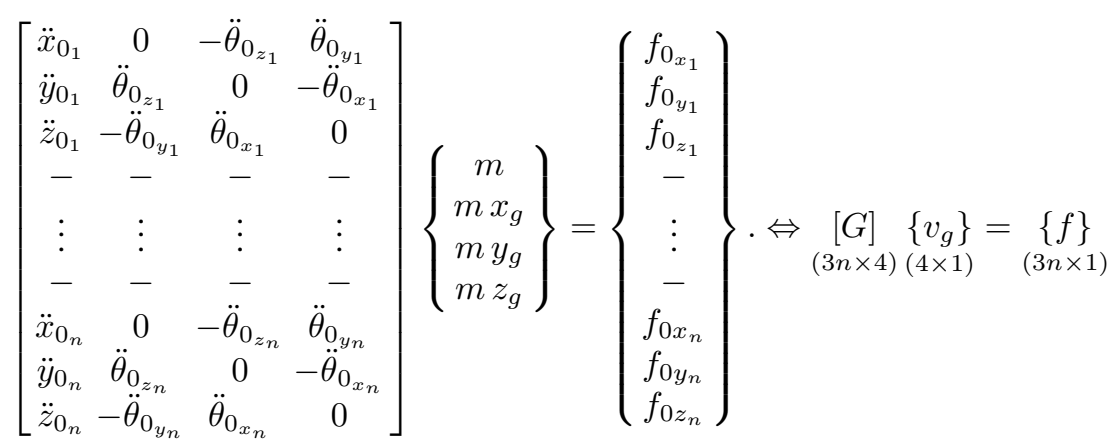


Where $\left\{v_{g}\right\}$ contains the four unknowns related to the force vector $\{f\}$ by an acceleration matrix $[G]$. So the mass and the centre of mass co-ordinates can be obtained through:

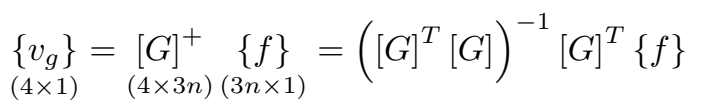

After locating the centre of mass coordinates, it is possible to carry out a subsequent step that can lead to the determination of the remaining six inertia properties, i.e, the moments and products of inertia.

\subsubsection{Second step}

The moments $M_{g_{x_{j}}}, M_{g_{y_{j}}}, M_{g_{z_{j}}}$ due to each excitation force, acting on point $j$, can be obtained as:

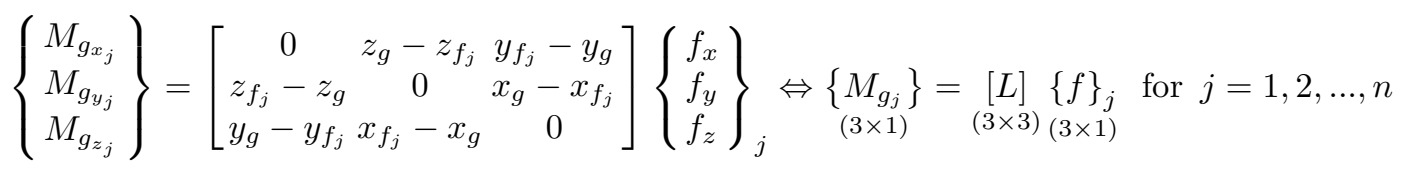

where $[L]$ is a transformation matrix. A final vector $\left\{U_{g}\right\}$ can be built from various vectors $\left\{M_{g_{j}}\right\}$ and the following relationship can be easily established:

$$
\left\{\begin{array}{c}
M_{g_{x_{1}}} \\
M_{g_{y_{1}}} \\
M_{g_{z_{1}}} \\
- \\
\vdots \\
- \\
M_{g_{x_{n}}} \\
M_{g_{y_{n}}} \\
M_{g_{z_{n}}}
\end{array}\right\}=\left[\begin{array}{cccccc}
\ddot{\theta}_{x_{1}} & 0 & 0 & -\ddot{\theta}_{g_{y_{1}}} & 0 & -\ddot{\theta}_{g_{z_{1}}} \\
0 & \ddot{\theta}_{g_{y_{1}}} & 0 & -\ddot{\theta}_{g_{x_{1}}} & -\ddot{\theta}_{g_{z_{1}}} & 0 \\
0 & 0 & \ddot{\theta}_{g_{z_{1}}} & 0 & -\ddot{\theta}_{g_{y_{1}}} & -\ddot{\theta}_{g_{x_{1}}} \\
- & - & - & - & - & - \\
\vdots & \vdots & \vdots & \vdots & \vdots & \vdots \\
- & - & - & - & - & - \\
\ddot{\theta}_{g_{x_{n}}} & 0 & 0 & -\ddot{\theta}_{g_{y_{n}}} & 0 & -\ddot{\theta}_{g_{z_{n}}} \\
0 & \ddot{\theta}_{g_{y_{n}}} & 0 & -\ddot{\theta}_{g_{x_{n}}} & -\ddot{\theta}_{g_{z_{n}}} & 0 \\
0 & 0 & \ddot{\theta}_{g_{z_{n}}} & 0 & -\ddot{\theta}_{g_{y_{n}}} & -\ddot{\theta}_{g_{x_{n}}}
\end{array}\right]\left\{\begin{array}{c}
J_{g_{x x}} \\
J_{g_{x y}} \\
J_{g_{x z}} \\
J_{g_{y y}} \\
J_{g_{y z}} \\
J_{g_{z z}}
\end{array}\right\} \Leftrightarrow\left\{\begin{array}{l}
\{(3 n \times 1) \\
(3 n \times 6)(6 \times 1) \\
\\
\end{array}\right.
$$

Where the vector $\left\{J_{g}\right\}$ contains the three inertia moments and the three inertia products, which can be obtained by calculating the pseudo-inverse $\left(^{+}\right)$of the acceleration matrix $[A]$ :

$$
\left\{J_{g}\right\}=[A]^{+}\left\{U_{g}\right\}=\left([A]^{T}[A]\right)^{-1}[A]^{T}\left\{U_{g}\right\}
$$

The determination of all the ten inertia properties is only possible if some numerical conditions are observed on the use of this type of methods; Lee et al. [8] have proven that at least three excitation forces should be applied on the structure and that, at least, three tri-axial measured points have to be considered.

A priori it sounds logical that results should improve whenever the number of applied forces increases. However, in this case, it is more important the way forces are applied, i.e., their position and direction, rather than the number of forces itself, as this aspect has a direct consequence in a better excitation of the rigid body motion and therefore in the quality of the results.

\subsection{Modal Methods (MM)}

The MM methods developed by Conti and Bretl [14] and Toivola and Nuutila [15] make use of the measured receptance responses due to the excitation applied in various points and directions. These methods require as many exciter locations/directions as needed to significantly excite all the six rigid body modes. In numerical terms, one excitation may be enough, in contrast with the minimum three excitations required by the IRM methods. The measured FRFs for each excitation condition are used to extract the modal properties (natural frequencies, damping ratios and mode shapes), using appropriate identification methods. The mass matrix $\left[M_{0}\right]$ related to the origin can thus be obtained using the orhogonality properties of the mass-normalised mode shape matrix $\left[\phi_{0}\right]$.

$$
\left[M_{0}\right]=\left[\phi_{0}\right]^{-T}\left[\phi_{0}\right]^{-1}
$$


These mode shapes can be obtained from the identified ones with respect to the physical co-ordinates $[\phi]$.

$$
\left[\phi_{0}\right]=\left(\left[R_{0}\right]^{T}\left[R_{0}\right]\right)^{-1}\left[R_{0}\right]^{T}[\phi]
$$

where $\left[R_{0}\right]$ represents the transformation matrix of the rigid body modes related to the $n$ tri-axial accelerometers [24]. The works $[8,24]$ proved that a minimum of three tri-axial measurement points are needed to evaluate $\left(\left[R_{0}\right]^{T}\left[R_{0}\right]\right)^{-1}$ and better results could be obtained when the three accelerometers formed a regular triangle. The mass of the body can be calculated as the average of the first three diagonal terms of $\left[M_{0}\right]$. Equating the non-zero elements of the upper right quadrant of $\left[M_{0}\right]$ matrix, to the same elements in mass matrix referred to the centre of mass, six equations are obtained [15,24].

Conti and Bretl [14] state that all the six rigid body modes must be identified prior to the estimation of the mass matrix. However, in practical situations there might be difficulties in exciting or identifying some of the rigid body modes. Toivola and Nuutila [15] solve the problem, as only 21 independent orthogonality conditions are required i.e., only four modes are needed. With this method only one mass value is obtained. The components of the inertia tensor are referred to the origin of the system of co-ordinates and should be transformed to the centre of mass using the co-ordinate transformation, as presented in [24].

\subsection{Direct Physical Parameter Identification Method (DPPIM)}

The DPPIM methods make use, like the previous, of the measured receptance responses due to the excitation applied in various points and directions. Huang and Lallement [13] propose a method for independent identification of the three types of parameters, mass, stiffness and damping matrices. Such a separation reduces the number of unknown parameters and thus can improve the numerical conditioning of the system. The formulation of this method leads to the following set of equations:

$$
\begin{aligned}
& {\left[Y_{1}\right]^{T}\left[K_{0}\right]\left[Y_{2}\right]-\left[S_{1}\right]\left[Y_{1}\right]^{T}\left[M_{0}\right]\left[Y_{2}\right]\left[S_{2}\right]=\left[\alpha_{i j}\right]} \\
& {\left[\bar{Y}_{1}\right]^{T}\left[K_{0}\right]\left[Y_{2}\right]-\left[\bar{S}_{1}\right]\left[\bar{Y}_{1}\right]^{T}\left[M_{0}\right]\left[Y_{2}\right]\left[S_{2}\right]=\left[\beta_{i j}\right]}
\end{aligned}
$$

where $\left[S_{1}\right]=\operatorname{diag}\left(\mathrm{i} \omega_{i}^{(1)}\right) \in C^{m, m}$ and $\left[S_{2}\right]=\operatorname{diag}\left(\mathrm{i} \omega_{j}^{(2)}\right) \in C^{n, n}$ are diagonal matrices of the chosen frequencies taken before $(m)$ and after $(n)$ each natural rigid body frequency, respectively. $\left[Y_{1}\right] \in C^{6, m}$ and $\left[Y_{2}\right] \in C^{6, n}$ are formed by the responses of the first and second series frequencies. $\left[\alpha_{i j}\right] \in C^{m, n}$ and $\left[\beta_{i j}\right] \in C^{m, n}$ are built as shown in [13]. Eliminating $\left[K_{0}\right]$ between Eqs (10) and (11) leads to:

$$
\left(\left[Y_{1}\right]^{T}\left(\left[\bar{Y}_{1}\right]^{T}\right)^{+}\left[\bar{S}_{1}\right]\left[\bar{Y}_{1}\right]^{T}-\left[S_{1}\right]\left[Y_{1}\right]^{T}\right)\left[M_{0}\right]\left[Y_{2}\right]\left[S_{2}\right]=\left[\alpha_{i j}\right]-\left[Y_{1}\right]^{T}\left(\left[\bar{Y}_{1}\right]^{T}\right)^{+}\left[\beta_{i j}\right]
$$

Separating Eq. (12) into its real and imaginary parts, a linear system of equations in the unknown ten inertia properties is obtained.

\section{Experimental case-study}

\subsection{Theoretical properties}

The first experimental case study is illustrated in Fig. 1, with the corresponding origin of the system of co-ordinates and the location of the tri-axial accelerometers. The theoretical inertia parameters of the actual tested structure were obtained by the SolidWorks modulation software; these values are assumed as correct for the sake of comparison. As it can be observed, the structure presents a high degree of symmetry that causes small values for the products of inertia relative to the selected system of axes. With the objective of observing the influence of symmetry properties on the obtained results the first structure was modified through the coupling of an additional sub-structure represented in Fig. 2. The modified structure was also tested in order to assess the unsymmetrical distribution of mass over the axes of reference. 


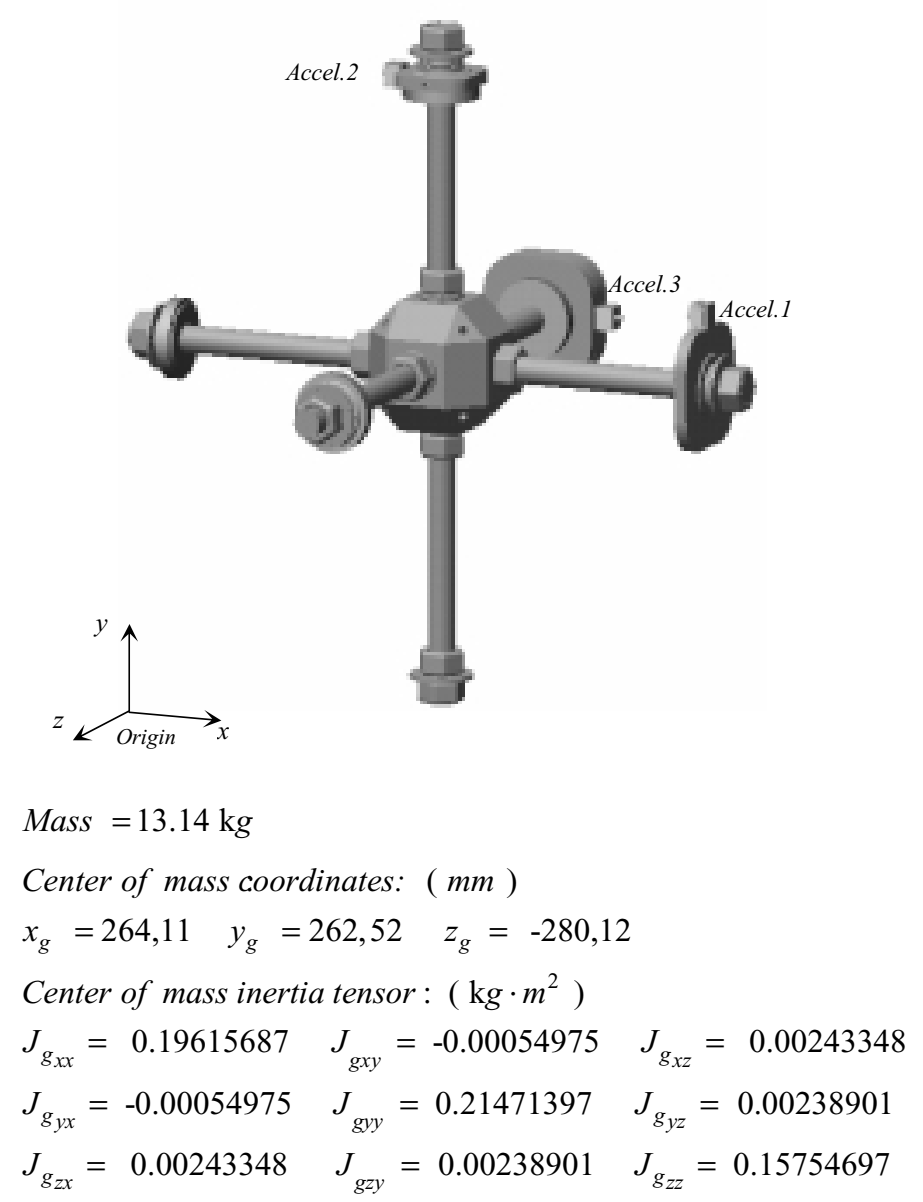

Fig. 1. Case study 1 (Structure I).

\subsection{Experimental set-up and data assessment}

\subsubsection{Suspension set-up}

Two different suspensions set-ups were implemented for each structure. For the IRM the suspension (Figs 3(a) and (b)) tries to simulate the freely supported conditions, required by this method. This set-up will lead to the concentration of all the rigid body modes at nearly zero Hz. The first flexible mode was observed to be distant enough from the last rigid body mode. In the other two methods, the DPPIM and MM, the suspension was built-up in order to allow the observation of a rigid body behaviour in the frequency range $0-12.5 \mathrm{~Hz}$. This will enable the observation of the rigid body modes of the suspended structure (a flexible response of the structure itself will occur above $100 \mathrm{~Hz}$ ). The set of springs was arranged in such a way to provide adequate axial and torsional stiffnesses which, combined with the inertia properties, allows the occurrence of all the rigid body modes in the selected frequency range $0-12.5 \mathrm{~Hz}$. The application of these two methods requires that the modes are separated enough, mainly in the case of DPPIM, where it is necessary to select two responses corresponding to two frequencies before and after each resonance. The softly support conditions were the same in both structures (Figs 3(c) and (d)). The stiffness of the used springs varies between 0.187 and $1.09 \mathrm{~N} / \mathrm{mm}$.

\subsubsection{Applied forces and measurement points}

Figure 4 shows the different applied forces on Structures I and II, used in IRM and DPPIM methods; these forces can be grouped according to the sets shown in Fig. 4. The measurement points are the same for both structures and 


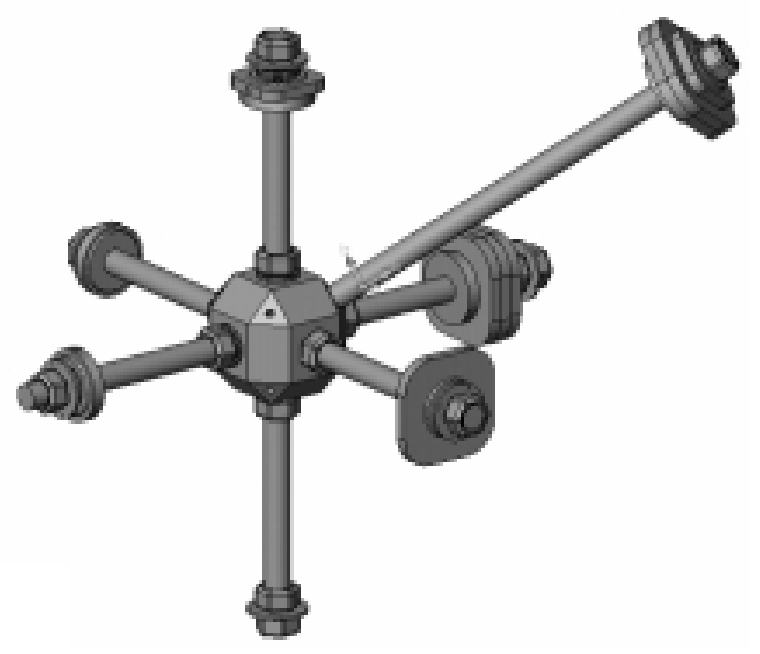

$$
\begin{aligned}
& \text { Mass }=15.69 \mathrm{~kg} \\
& \text { Center of mass coordinates: ( } \mathrm{mm} \text { ) } \\
& x_{g}=295,75 \quad y_{g}=294,17 \quad z_{g}=-308,13 \\
& \text { Center of mass inertia tensor: }\left(\mathrm{kg} \cdot \mathrm{m}^{2}\right) \\
& J_{g_{x x}}=0.35908174 \quad J_{g x y}=0.08875010 \quad J_{g_{x z}}=-0.07757822 \\
& J_{g_{y x}}=0.08875010 \quad J_{g y y}=0.37750727 \quad J_{g_{y z}}=-0.07768094 \\
& J_{g_{z x}}=-0.07757822 \quad J_{g z y}=-0.07768094 \quad J_{g_{z z}}=0.33786348
\end{aligned}
$$

Fig. 2. Case study 2 (Structure II).
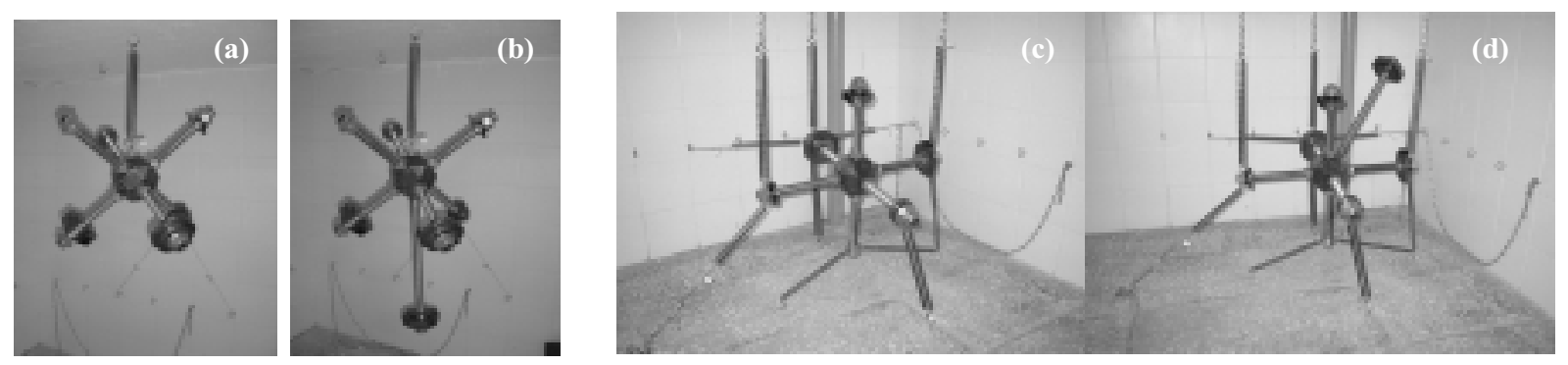

Fig. 3. Suspension set-ups used for the application of: IRM (a),(b) and MM and DPPIM methods (c),(d).

are presented in Fig. 1.

For the application of MM methods three measurement points have been selected and three excitation forces have been applied; a necessary condition for the identification of the rigid body modal properties is that at least one of the forces acts in one of the accelerometer directions. The origin of the co-ordinate system corresponds to the location of accelerometer 2, according to the recommendation given in [11], Figs 5 and 6.

All the forces have been applied through an impact hammer, with a rubber tip to obtain the receptance FRFs in the range of the rigid body modes $(0-12.5 \mathrm{~Hz})$ needed for the application of DPPIM and MM and with a hard plastic tip to obtain the accelerance FRFs in the range of $0-200 \mathrm{~Hz}$ (that encompasses the first flexible modes), for the IRM. 


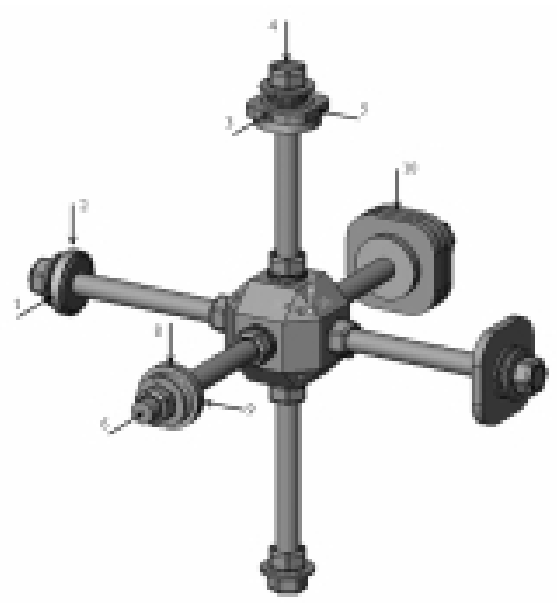

\begin{tabular}{|c|c|c|}
\hline Sets & Pestal & Fantim \\
\hline \multirow{3}{*}{ I } & 2 & $\bar{y}$ \\
\hline & 8 & $y$ \\
\hline & 9 & $x$ \\
\hline \multirow{3}{*}{ II } & 5 & $x$ \\
\hline & 8 & $y$ \\
\hline & 9 & $\bar{x}$ \\
\hline \multirow{3}{*}{ III } & 3 & $z$ \\
\hline & 4 & $y$ \\
\hline & 5 & $x$ \\
\hline \multirow{3}{*}{ IV } & 4 & $y$ \\
\hline & 8 & $y$ \\
\hline & 10 & $y$ \\
\hline
\end{tabular}

\begin{tabular}{|c|c|c|}
\hline Sets & $P=n$ & Dention \\
\hline \multirow{3}{*}{ v } & 1 & $z$ \\
\hline & 5 & $x$ \\
\hline & Do & $z$ \\
\hline \multirow{3}{*}{ v1 } & 1 & $\frac{\pi}{2}$ \\
\hline & 2 & $y$ \\
\hline & 3 & $z$ \\
\hline \multirow{3}{*}{ VII } & 1 & $\frac{2}{2}$ \\
\hline & 2 & $\bar{y}$ \\
\hline & B & $y$ \\
\hline \multirow{3}{*}{ VIII } & 1 & $z$ \\
\hline & 3 & 7 \\
\hline & 5 & \\
\hline
\end{tabular}

Fig. 4. Applied forces for the application of IRM and DPPIM methods, Structures I and II.

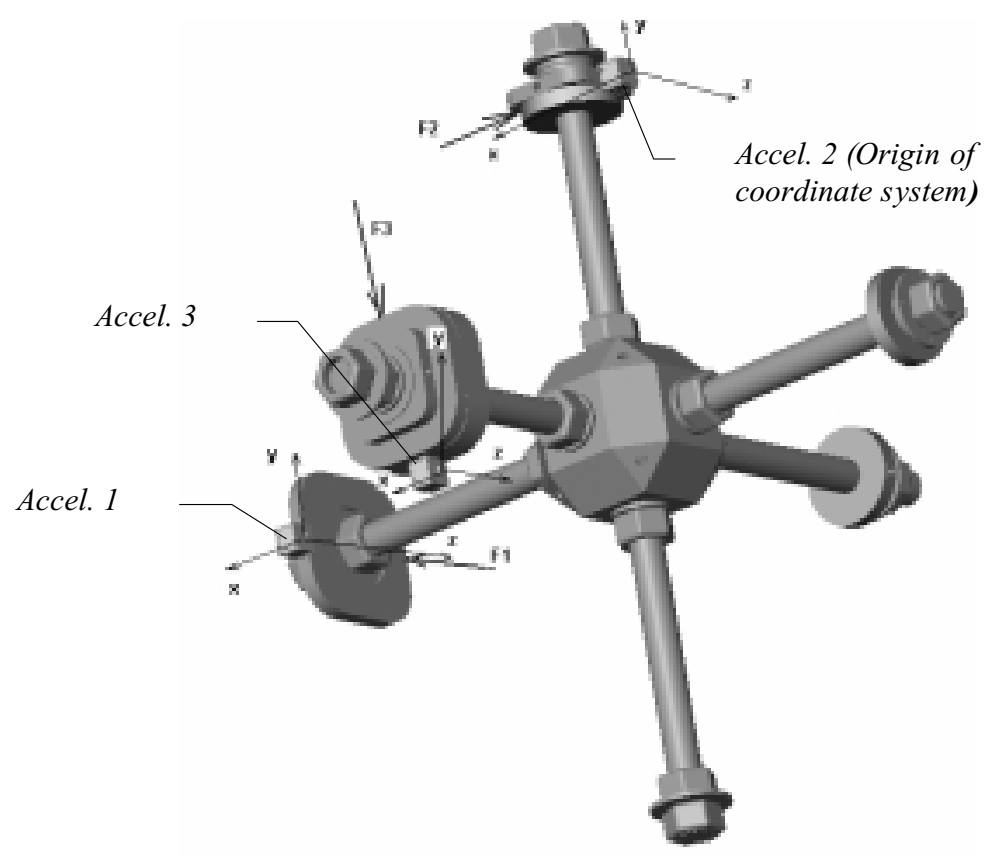

Fig. 5. Applied forces and chosen origin for the application of MM methods to Structure I.

\section{Results}

\subsection{Application of IRM and DPPIM}

The application of IRM required (for each set of applied forces) nine accelerance FRFs to be collected w.r.t the measuring co-ordinate system. Subsequently, a modal identification was performed on all FRFs to obtain the mass line values and then a co-ordinate transformation led to the six accelerations $\left(\ddot{x}_{0}, \ddot{y}_{0}, \ddot{z}_{0}, \ddot{\theta}_{0 x}, \ddot{\theta}_{0 y}, \ddot{\theta}_{0 z}\right)$ at the origin.

For the application of DPPIM and for each set of applied forces, nine responses have been collected w.r.t the measuring co-ordinate system. A subsequent transformation led to the six responses $\left(x_{0}, y_{0}, z_{0}, \theta_{0 x}, \theta_{0 y}, \theta_{0 z}\right)$ at the origin. The Norm Indicator tool (NI) [11] was then applied as an auxiliary tool to identify the excitation level 


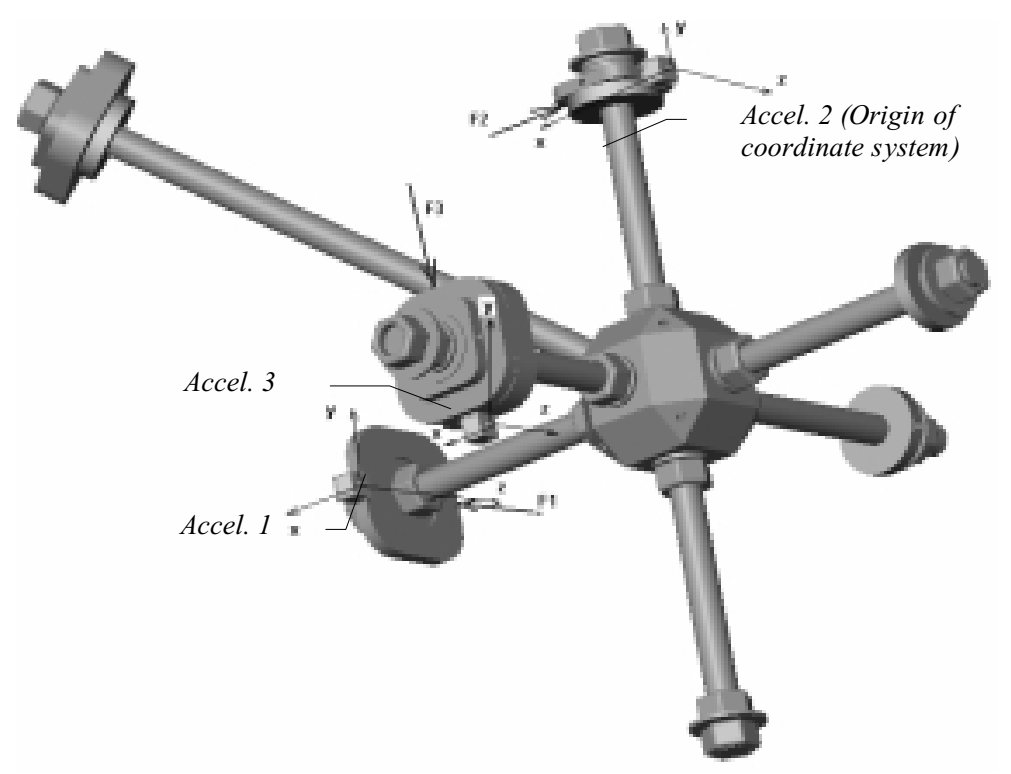

Fig. 6. Applied forces and chosen origin for the application of MM methods to Structure II.
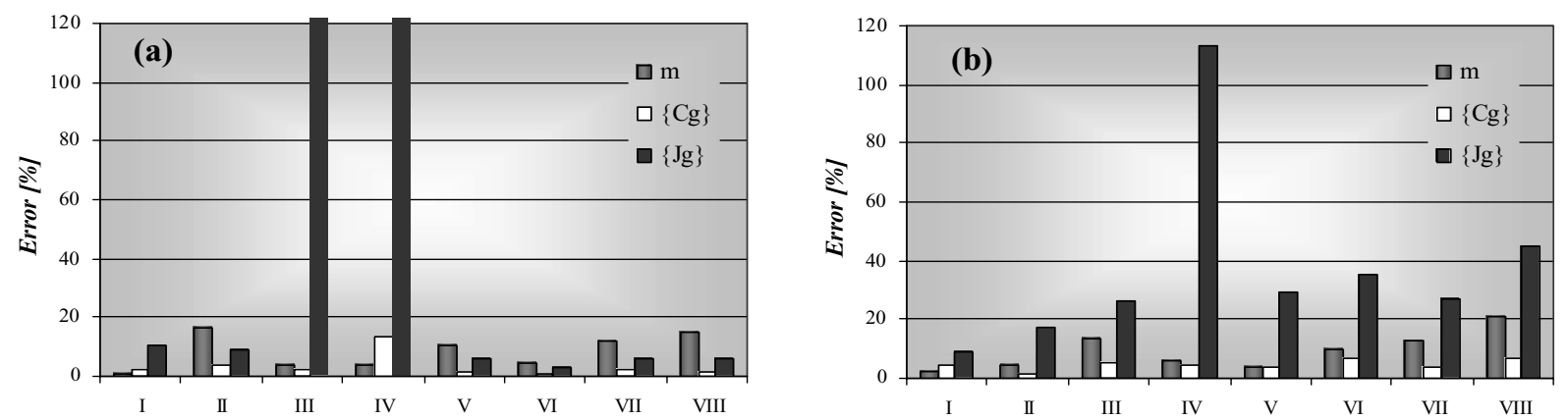

Fig. 7. Results obtained with the application of the (a) IRM and (b) DPPIM for each set of applied forces on Structure I.

of the rigid body modes and the corresponding natural frequencies. The values of the displacements and rotations occurring at the two frequencies immediately before and after each rigid body natural frequency were then used. The relative errors for the mass, vector of centre of mass co-ordinates $\left\{C_{g}\right\}$ and vector of moments and products of inertia $\left\{J_{g}\right\}$ as well as the summation of all the parameter errors were then obtained for both methods.

\subsubsection{Remarks}

It can be observed that, in general, the IRM method gives better results than the DPPIM. The only exceptions happen with the sets of forces III and IV, as it can be observed in Figs 7-10. These sets were previously identified, as critical sets, in the works [8,24]. In these works it is additionally demonstrated that a minimum of three forces and three responses are necessary to obtain the numerical solution. The quality of the results obtained with the application of both IRM and DPPIM methods decreases either when the FRFs present high levels of noise or when the rigid body modes are not excited or low excited [24].

\subsection{Application of MM methods}

After obtaining all of the nine receptance FRFs, for each excitation force, the Norm Indicator (NI), developed by the authors, was applied. This indicator provides information about the rigid body natural frequencies and about the 


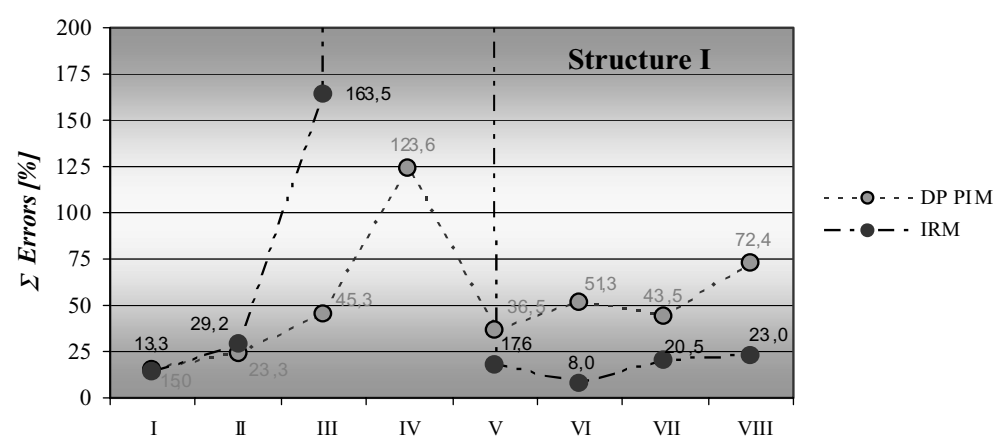

Fig. 8. Total error for each set of applied forces on Structure I, applying of the IRM and DPPIM.
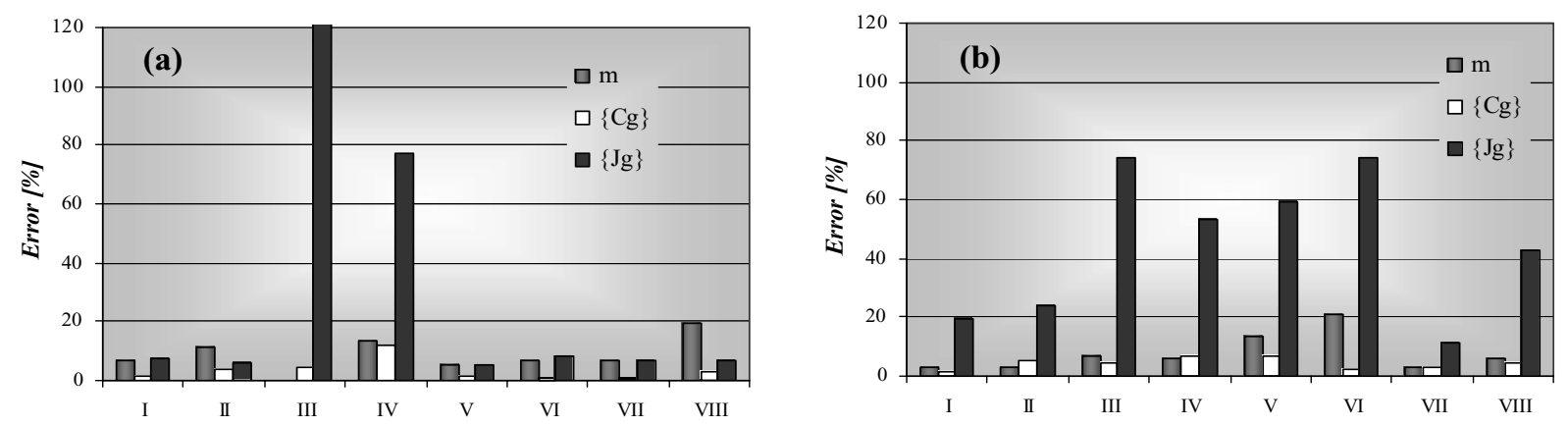

Fig. 9. Results obtained with the application of the (a) IRM and (b) DPPIM for each set of applied forces on Structure II.

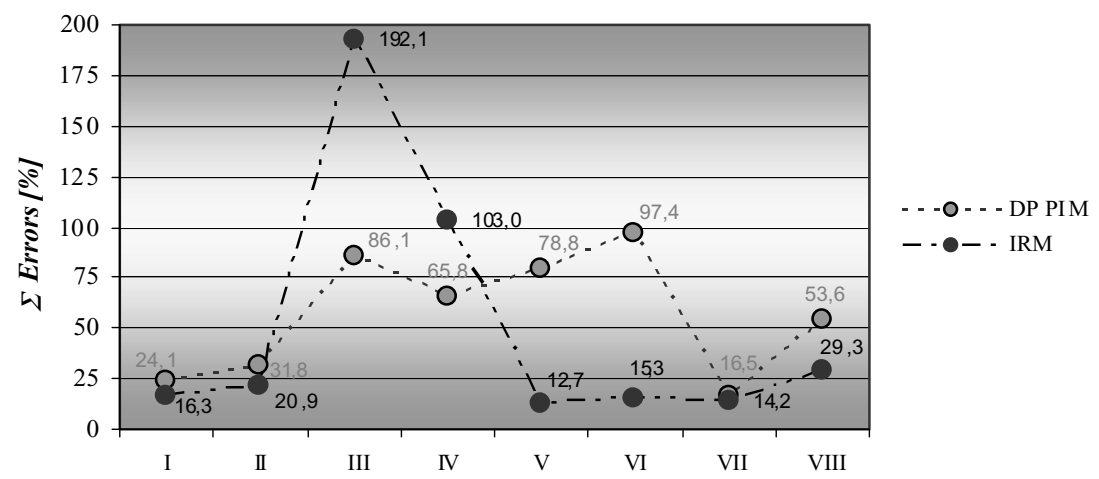

Fig. 10. Total error for each set of applied forces on Structure II applying of the IRM and DPPIM.

level of excitation of each rigid body mode (Fig. 11). For the identification of the modal parameters, a Single Input Multiple Output (SIMO) modal analysis was performed using MODENT [27].

From Fig. 11 it can be observed that there are situations where some forces excite more certain modes than others. It was concluded in $[11,18,24]$ that the accuracy of the results increases when the rigid body modes are better excited. So, with the information given by the NI and the identified rigid body modal properties obtained with different forces, a refined modal matrix can be constructed with all the best excited rigid body modes:

The relative errors for the mass, vector of centre of mass co-ordinates $\left\{C_{g}\right\}$ and vector of moments and products of inertia $\left\{J_{g}\right\}$ as well as the summation of all the parameter errors are presented in Figs 12 and 13, respectively: 

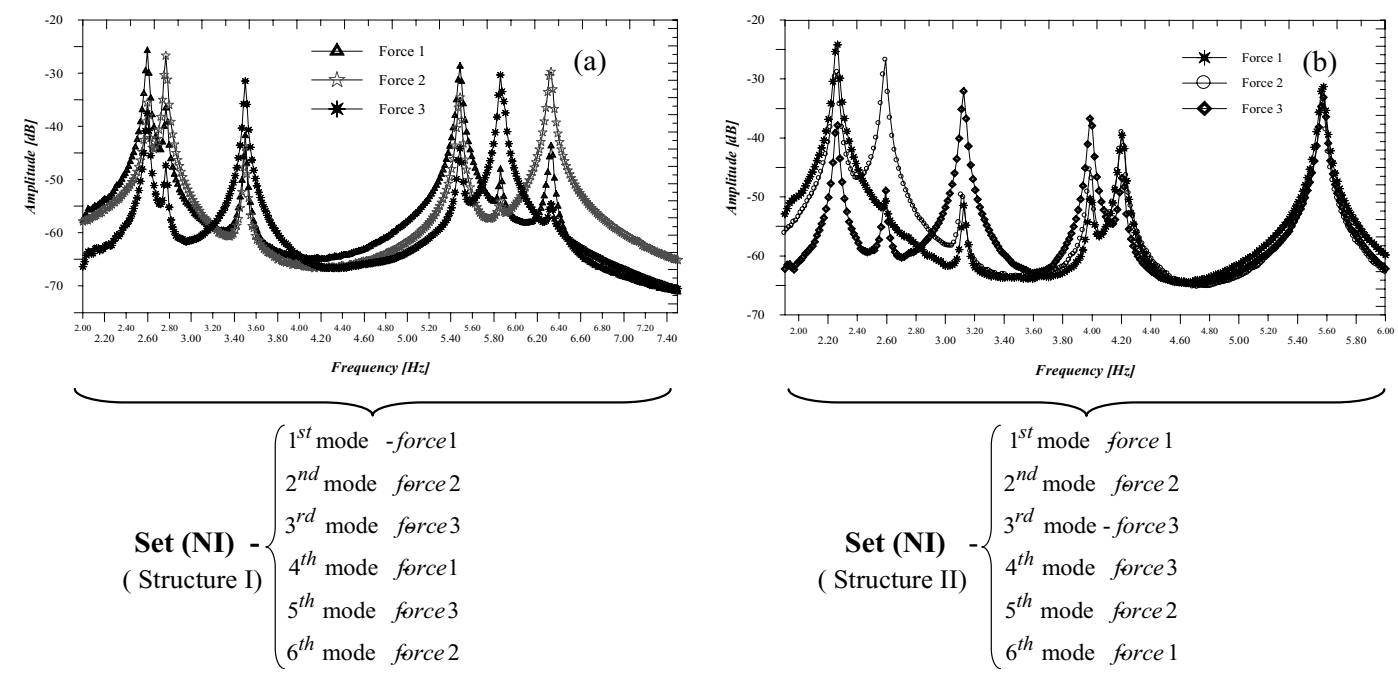

Fig. 11. Results obtained with the Norm Indicator (NI) for each applied force on: (a) structure I and (b) structure II.
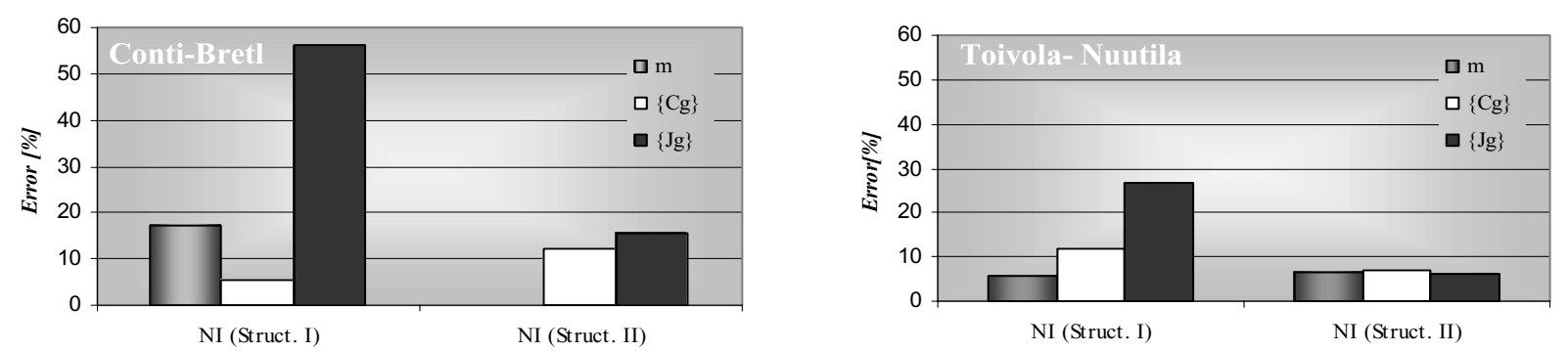

Fig. 12. Results obtained with Conti-Bretl and Toivola-Nuutila methods, using the refined modal matrices constructed with information given by the NI.

\subsubsection{Remarks}

From Figs 12 and 13 it can be observed that Toivola-Nuutila method gives, in general, better results than ContiBretl method, independently of the structure in study. The same conclusion was obtained in a previous work carried out by the authors, using another structure [24, 26].

Another conclusion is that for rigid bodies that present high symmetry relatively to the chosen system of coordinates, sometimes it is not easy to excite all the rigid body modes with only one force as it is shown in Fig. 11. In this case it is advisable to use information resulting from several forces in conjunction with the Norm Indicator, as it is proven in $[11,18,24,26]$.

\section{Conclusions}

The three different types of methods studied to obtain the rigid body properties allow to obtain good results if the restrictions of each method are observed and if the measured FRFs have good quality.

Within the MM methods, Toivola-Nuutila's leads to better results than the Conti-Bretl method. These results can be further improved by taking into account the best excited rigid body modes obtained from different sets of forces. The Norm Indicator is a crucial tool for the construction of the refined modal matrix.

The DPPIM is easier to handle than the other two methods because it does not require the application of modal identification techniques to the measured FRFs. It uses directly the raw available FRF data.

A definite conclusion on the best method is not possible, as it is always case dependent; it is advisable to use more than one method to compare the results. 


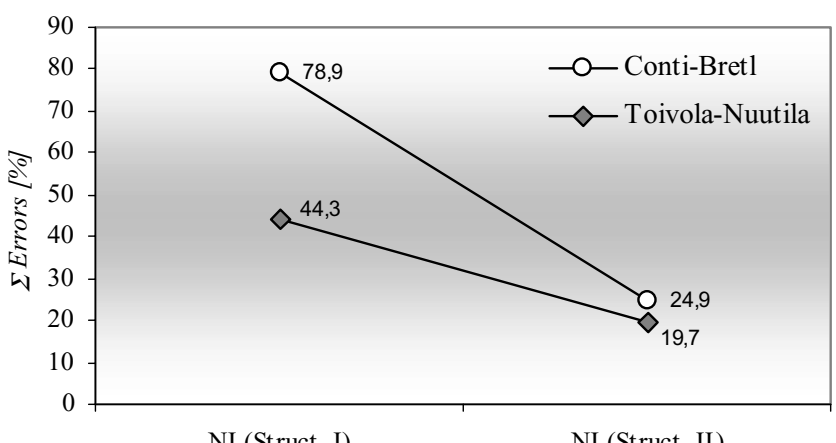

Fig. 13. Summation of all the parameter errors, with Conti-Bretl and Toivola-Nuutila methods, using the refined modal matrices constructed with information given by the NI.

\section{Acknowledgement}

The current investigation had the support of FCT, under the project POCTI2010.

\section{References}

[1] C. Schedlinski and M. Link, A Survey of Current Inertia Parameter Identification Methods, Mechanical Systems and Signal Processing 15(1) (2001), 189-211.

[2] S.M. Pandit and Z.-Q. Hu, Determination of Rigid Body Characteristics from Time Domain Modal Test Data, Journal of Sound and Vibration 177(1) (1994), 31-41.

[3] H. Hahn, Inertia Parameter Identification of Rigid Bodies Using a Multi-Axis Test Facility, Proc. of the $3^{d}$ IEEE Conf. on Control Applications, 1994, 1735-1737.

[4] Y.S. Wei and J. Reis, Experimental Determination of Rigid Body Inertia Properties, Proc. of the th International Modal Analysis Conference (IMAC VII), Las Vegas, Nevada, USA, 1989, 603-606.

[5] U. Füllekrug and C. Schedlinski, Inertia Parameter Identification from Base Excitation Test Data, Proc. of the $5^{\text {th }}$ International Symposium on Environmental Testing for Space Programmes, Noordwijk, Netherlands, 2004.

[6] A. Fregolent and A. Sestieri, Identification of Rigid Body Inertia Properties from Experimental Data, Mechanical Systems and Signal Processing 10(6) (1996), 697-709.

[7] A.P.V. Urgueira, On the Rigid Body Properties Estimation from Modal Testing, Proc. of the $13^{\text {th }}$ International Modal Analysis Conference (IMAC XIII), Nashville, Tennessee, USA, 1995, 1479-1483.

[8] H. Lee, Y. Park and Y. Lee, Response and Excitation Points Selection for Accurate Rigid Body Inertia Properties Identification, Mechanical Systems and Signal Processing 1(4) (1999), 571-592.

[9] A.P.V. Urgueira and R.A.B. Almeida, Dynamic Properties of Rigid Body Systems from Vibration Measurement, Proc. of the International Conference on Structural Dynamics Modelling, Madeira, Portugal, 2002, 211-215.

[10] J.A. Mangus, C. Passarello and C. Vankarsen, Estimation Rigid Body Properties from Frequency Reaction Measurements, Proc. of the $11^{\text {th }}$ International Modal Analysis Conference (IMAC XI), Kissimmee, Florida, USA, 1993, 469-472.

[11] R.A.B. Almeida, A.P.V. Urgueira and N.M.M. Maia, Evaluation of Rigid Body Properties from Frequency Response Data, Proc. of the International Conference on Modal Analysis Noise and Vibration Engineering (ISMA 2004), Leuven, Belgium, $2004,2409-2423$.

[12] N. Wagner, Estimation of Rigid Body Parameters Using the Matrix Logarithm, http://www.mecha.unistuttgart.de/Mitarbeiter/Wagner/ papers/nmcm2002.pdf, 2002.

[13] S.J. Huang and G. Lallement, Direct Estimation of Rigid Body Properties from Harmonic Forced Responses, Proc. of the $15^{\ddagger h}$ International Modal Analysis Conference (IMAC XV), Orlando, Florida, USA, 1997, 175-180.

[14] P. Conti and J. Bretl, Mount Stiffness and Inertia Properties from Modal Test Data, Journal of Vibration, Acoustics, Stress and Reliability in Design 111 (1989), 134-138.

[15] J. Toivola and O. Nuutila, Comparison of Three Methods for Determining Rigid Body Inertia Properties from Frequency Response Functions, Proc. of the $11^{\text {th }}$ International Modal Analysis Conference (IMAC XI), Kissimmee, Florida. USA, $1993,1126-1132$.

[16] Q. Zeng and L. Zhang, Estimation of Rigid Body Modes and Support Properties, Proc. of the 8th International Modal Analysis Conference (IMAC VIII), Kissimmee, Florida, USA, 1990, 1116-1121.

[17] M. Okuma, T. Oho and Q. Shi, Development of Experimental Spatial Matrix Identification Method (Theory and Basic Verification with a Frame Structure), Journal of Sound and Vibration 219(1) (1999), 5-22.

[18] N.M.M. Maia, R.A.B. Almeida and A.P.V. Urgueira, New Developments on the Identification of Rigid Body Properties, Proc. of the International Congress of Noise and Vibration Emerging Methods (NOVEM 2005), San Raphael, France, 2005.

[19] R.A.B. Almeida, N.M.M. Maia and A.P.V. Urgueira, Identification of Rigid Body Properties from Vibration Measurements, Journal of Sound and Vibration 299(4-5) (2007), 884-899. 
[20] D. Jonhson, J.V. Karsen, J. Blough and M. Rao, Estimation of a Power train Inertia Properties via an In-situ Method, Proc. of the International Conference on Modal Analysis Noise and Vibration Engineering (ISMA 2006), Leuven, Belgium, 2006, pp. 2521-2535.

[21] J. Toivola and O. Nuutila, Comparison of Three Methods for Determining Rigid Body Inertia Properties from Frequency Response Functions, Proc. of the $11^{\text {th }}$ International Modal Analysis Conference (IMAC XI), Kissimmee, Florida. USA, 1993, 1126-1132.

[22] J.P.G. Martins, MSc Thesis (in Portuguese), "Identification of the Dynamic Properties of a Rigid Body, Department of Mechanical Engineering and Industrial, Faculty of Science and Technology, New University of Lisbon, Portugal, 1999.

[23] R.A.B. Almeida, N.M.M. Maia and A.P.V. Urgueira, Direct Method versus Modal Methods in the Identification of Rigid Body Properties, Proc. of the $30^{t h}$ International Congress on Sound and Vibration (ICSV13), Vienna, Austria, 2006.

[24] R. Almeida, PhD Thesis (in Portuguese), Evaluation of the Dynamic Characteristic of Rigid Bodies Based on Experimental Results, Department of Mechanical Engineering and Industrial, Faculty of Science and Technology, New University of Lisbon, Portugal, 2006.

[25] W. Leurs, M. Gielen, B. Bughmans, Dierckx, Calculation of Rigid Body Properties from FRF Data: Practical Implementation and Test Cases, LMS, 2000, 1998.

[26] N.M.M. Maia, R.A.B. Almeida and A.P.V. Urgueira, Modal Analysis in the Identification of Rigid Body Properties, Proc. of the $1 \mathrm{t}^{\text {th }}$ International Conference on Vibration Engineering, Timisoara, Romania, 2005.

[27] MODENT, Integrated Software for Structural Dynamic, Imperial College of Science Technology and Medicine, University of London: London, UK, ICATS 1988-2000. 

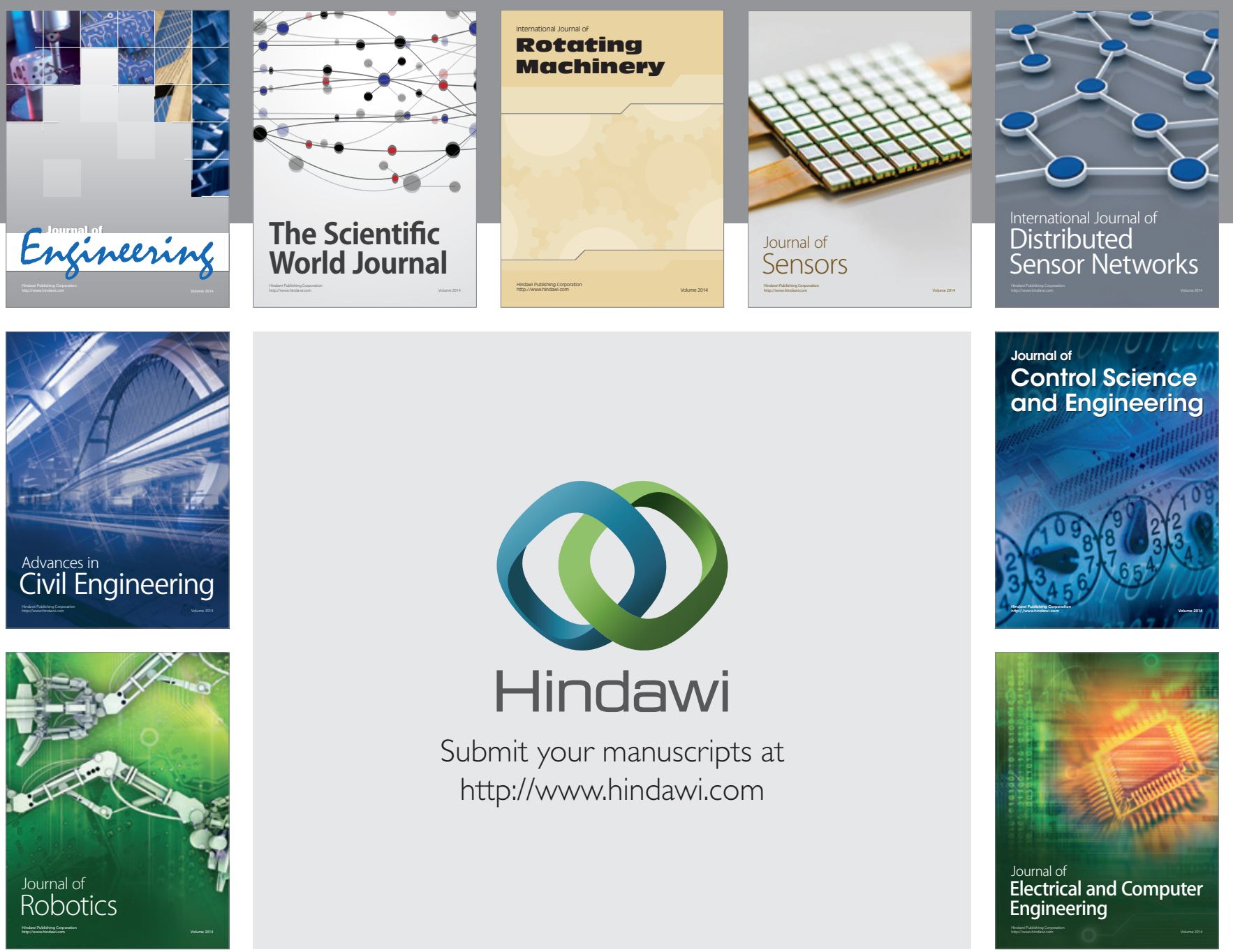

Submit your manuscripts at

http://www.hindawi.com
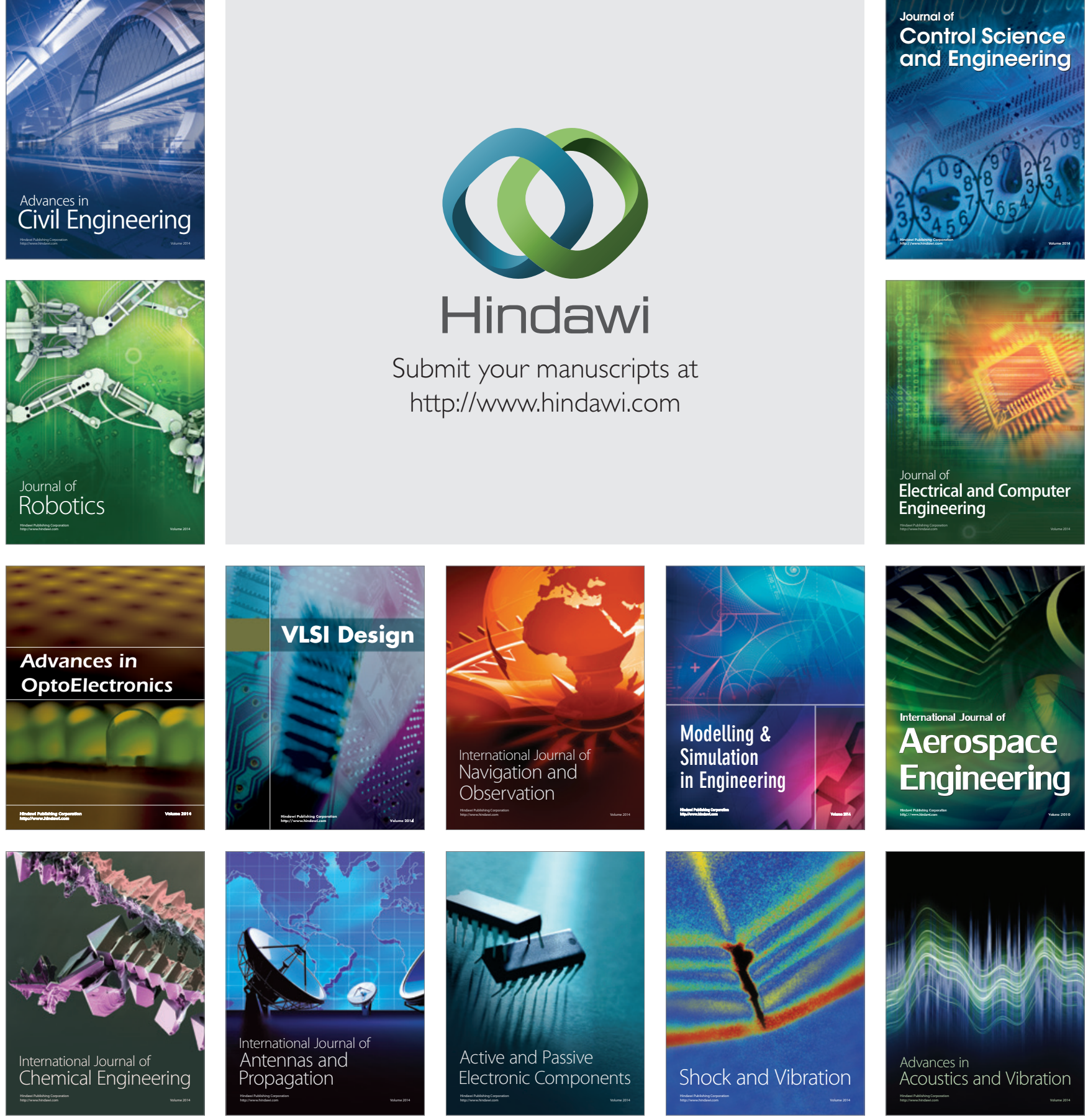